Rev. Psicol. (Arequipa. Univ. Catól. San Pablo) / Año 2021 / Vol 11 / N 2 / pp. 85-108 ISSN 2306-0565 versión impresa / ISSN 2311-7397 versión on line

\title{
«Sí, tiene usted razón: Hay que admirarse de adónde nos ha arrojado el destino»: Walter Blumenfeld (Lima, Perú) y Arthur Liebert (Belgrado, Yugoslavia) en el exilio
}

«Yes: You Are Right: We Have to Admire Where Destiny Has Thrown Us»:

Walter Blumenfeld (Lima, Peru) and Arthur Liebert

(Belgrade, Yugoslavia) in the Exile

\author{
Ramón León Donayre \\ Universidad Ricardo Palma, Lima, Perú \\ iD https://orcid.org/oooo-0oo-7380-6732 \\ Correspondencia: rld30850@yahoo.com.mx \\ Zdravko Kučinar \\ Universidad de Belgrado, Belgrado, Serbia \\ (iD https://orcid.org/oooo-0oo2-7065-68o \\ Correo electrónico: zkucinar@mts.rs
}

\section{Resumen}

Walter Blumenfeld (882-967) y Arthur Liebert (878-946) fueron dos de los numerosos intelectuales que se vieron obligados a dejar Alemania durante los años del nacionalsocialismo. El primero, psicólogo y profesor en la Universidad Técnica de Dresde, se estableció en el Perú desde 935 hasta el fin de sus días. El segundo, Liebert, profesor en la Universidad de Berlín y director de Kant-Studien y de la Kant-Gesellschaft, partió a Yugoslavia, donde permaneció entre 934 y 939, para de allí trasladarse a Inglaterra. La presente comunicación trata de la correspondencia entre ambos (936-939), que permite conocer las dificultades experimentadas por ellos en sus nuevos lugares de residencia, así como el trabajo que desplegó Liebert en la fundación y edición de Philosophia, revista que puede ser considerada la más importante del exilio alemán.

Palabras clave: Walter Blumenfeld, Arthur Liebert, historia de la psicología.

\section{Abstract}

Walter Blumenfeld (882-967) and Arthur Liebert (878-946) were two of the many intellectuals who were forced to leave Germany during the years of National Socialism. The first, a psychologist and professor at the Technical University of 
Dresden, settled in Peru from 935 to the end of his days. The second, Liebert, a professor at the University of Berlin and director of Kant-Studien and the KantSociety, left for Yugoslavia, where he stayed between 934 and 939, and from there moved to England. This communication deals with the correspondence between the two (936-939), which reveals the difficulties experienced by them in their new places of residence, as well as the work carried out by Liebert in the foundation and edition of Philosophia, a journal that can be considered the most important of the German exile.

Keywords: Walter Blumenfeld, Arthur Liebert, history of psychology.

\section{Introducción}

«Sí, tiene Usted razón: hay que admirarse de adónde ha sido capaz el destino de arrojarnos»: así finaliza su carta del 26 de marzo de 937, de Arthur Liebert, en Belgrado, a Walter Blumenfeld, en Lima (Perú).

Entre Lima (Perú) y Belgrado (Serbia) hay una distancia de o mil kilómetros. Pero la geográfica no es la única distancia que separa a ambas ciudades: los idiomas en una y en otra son completamente diferentes, como también la cultura y la historia. Sin embargo, a mediados de los años '3o hubo una activa comunicación entre ambas urbes protagonizada por dos académicos alemanes obligados a emigrar ante la irrupción del nacionalsocialismo: Walter Blumenfeld (882-967) y Arthur Liebert (878-946).

Cada uno fue una personalidad destacada en su especialidad. Blumenfeld era un destacado psicólogo y, tras su forzada emigración al Perú, se convertiría en el pionero del enfoque experimental de la psicología en ese país (Alarcón 2000; León 983). Liebert, por su parte, era un conocido filósofo neokantiano que, además de una significativa obra escrita, poseía cualidades de exitoso administrador académico demostradas por su gestión al frente de la Kant-Gesellschaft y de la revista Kant-Studien.

Como era frecuente entre los académicos alemanes de esa época, el horizonte intelectual de ambos se extendía más allá de sus respectivas especialidades: Blumenfeld tenía un gran interés por la filosofía y Liebert por la psicología y la pedagogía. Y, por último, ambos eran activos escritores de cartas. En una época sin internet ni whatsapp, las cartas eran la única forma de mantener activos los vínculos familiares, amicales y profesionales, de estar al tanto de lo que ocurría en Alemania, y también, de combatir la nostalgia.

En sus cartas se ponen de manifiesto la nostalgia, así como la mirada de desconcierto hacia la nueva realidad en que se encontraban, $\tan$ distante y distinta de la Alemania que se habían visto obligados a dejar. Pero también se revelan sus esfuerzos por superar la adversidad vía la prosecución de su trabajo académico y de sus proyectos de investigaciones y libros. Particularmente interesante y casi 
conmovedor es el caso de Liebert, que en medio de su difícil situación, fundó una sociedad académica y una revista.

La presente comunicación (basada en la correspondencia que sostuvieron entre 936 y 939 ) trata de sus actividades, cumplidas en ambientes, circunstancias, idiomas y entre personas que les eran extraños, e informa sobre sus impresiones acerca de la realidad que los rodeaba.

\section{El exilio}

Desde antes de llegar al poder los nacionalsocialistas predicaban el antisemitismo en Alemania. Adolfo Hitler, su líder, había publicado en 925 Mein Kampf (leída por miles, al menos en los primeros años después de su aparición; Kellerhoff, 205), en el que los lectores podían conocer sus actitudes radicales, si bien muchos imaginaban que en el hipotético caso de que llegara al poder estas se tornarían más racionales. Más aún si, como lo señala Le Rider (206), «el historiador se pregunta quién era judío en la Viena de fines de siglo» (p. 23), aludiendo al grado en el cual la población judía se encontraba integrada, por cierto no solo en la capital del Imperio Austro-Húngaro, sino también en Alemania.

Tras la muerte del presidente Hindenburg en 934, Hitler se hizo del poder total y puso en práctica radicales medidas antisemitas, sin mayores consideraciones de humanidad, de reconocimientos de méritos ni de racionalidad política. Así, lo que visionariamente relataban Lion Feuchtwanger (884-958; uno de los literatos más conocidos en ese entonces) en su novela Los hermanos Opperman (Feuchtwanger, 933), o Hugo Bettauer (882-925), en Die Stadt ohne Juden (Bettauer, 922), esto es maltratos a ciudadanos judíos, abierta discriminación y por último su eliminación, se volvió realidad.

No solo los grandes y pequeños empresarios y comerciantes así como profesionales, fueron afectados por el antisemitismo que se desató bajo el régimen nazi. También lo fueron los profesores universitarios. El $7 \mathrm{de}$ abril de 933 el régimen nacionalsocialista emitió el Gesetz zur Wiederherstellung des Berufsbeamtemtums (Ley del Restablecimiento del Servicio Civil) (Reichgesetzblatt, 933), mediante el cual todos aquellos que no fueran de origen ario debían ser retirados de sus funciones en el estado o enviados al retiro forzoso. La norma estaba en realidad dirigida a funcionarios judíos, pues el reglamento de esa ley especificaba que no eran considerados arios quienes tuvieran padres o abuelos judíos, bastando que solo uno de los progenitores o abuelos hubiera pertenecido a la religión judía.

Esta norma afectó a muchísimos académicos en todas las ramas del saber. Economistas, médicos, abogados, filósofos, ingenieros, lingüistas, muchos de ellos de renombre internacional, se vieron de un día para otro privados de su posición laboral en las universidades, y sometidos a una serie de normas discriminatorias (véase Böhne \& MotzkauValeton, 992; Heilbut, 983; Fermi, 97; Fleck, 205; una exhaustiva relación de intelectuales y científicos emigrados 
se encuentra en Strauss \& Röder, 983). Algunos tuvieron que pasar por mil peripecias para lograr una nueva posición profesional en el exterior (tal el caso del neurólogo Kurt Goldstein (Benzenhöfer \& Hack-Molitor, 207); otros fracasaron en el intento y se suicidaron o fueron asesinados en los campos de concentración (Leff, 209). Entre los muchos afectados se encontraban Walter Blumenfeld y Arthur Liebert, judío.

\section{Walter Blumenfeld}

Walter Blumenfeld había sido hasta su separación, profesor de la Technische Hochschule zu Dresden (la Universidad Técnica de Dresde), llegando a alcanzar la categoría de Ausserördentlicher Professor. Tras estudiar ingeniería y graduarse en 906, ingresó a trabajar como ingeniero electricista en la Allgemeine-ElektrizitätsGesellschaft, en Berlín. En 908, gracias al apoyo financiero de su padre volvió a las aulas universitarias, esta vez para estudiar (hasta 93) psicología y filosofía en la Universidad de Berlín.

Doctorado en psicología con un trabajo sobre psicofísica de la visión bajo la dirección de Carl Stumpf (848-936) (Blumenfeld, 93), cumplió servicio militar durante los años de la Primera Guerra Mundial y después se incorporó a la mencionada Technische Hochschule desplegando una permanente actividad investigatoria expresada no solo en artículos en el campo de la psicofísica sino también en el de la psicotécnica y la epistemología de la psicología (e.g. Blumenfeld, 920, 923, 925a, 925b, 93a). Asimismo, dio a la luz dos libros dedicados a temas que hoy serían considerados como propios de la psicología cognitiva (Blumenfeld, 93b, 933).

Separado de sus funciones docentes, Blumenfeld emprendió una infructuosa búsqueda de una nueva posición laboral en Europa. Finalmente, en 935 aceptó una oferta desde el lejano Perú para desempeñarse como profesor en la Universidad Nacional Mayor de San Marcos, en la capital peruana, que además le ofrecía el cargo de director del recién creado Instituto de Psicología y Psicotécnica.

El Perú era un país que Blumenfeld no conocía y cuya lengua y cultura le eran asimismo extrañas. Sin embargo, decidió aceptar la oferta que se le había hecho y en 935 se hizo rumbo a su nuevo destino. Debió pensar quizás que su estancia en el país sudamericano sería provisoria y en la posibilidad de un retorno a Alemania tan pronto el régimen de Hitler se derrumbara por acción de una mayoría parlamentaria preocupada por devolver a su país al ámbito de la democracia.

Eso no sucedió. Blumenfeld permanecería el resto de su vida en el país andino. Sus años en el Perú, especialmente los primeros, estuvieron caracterizados por numerosas dificultades. Como hemos dicho, el idioma le era desconocido, pero prontamente llegó a familiarizarse con él y tras algún tiempo reinició sus publicaciones, esta vez en castellano. Su obra más importante en la psicología, Introducción a la psicología experimental (Blumenfeld, 
946), fue publicada en castellano y en el Perú.

No solo el idioma era un problema para el emigrante alemán. Las universidades peruanas padecían de una gran limitación de recursos, con bibliotecas muy mal abastecidas, presupuestos reducidos y la intervención de factores políticos. Todo esto hacía muy difícil la labor de investigación.

Por si todo esto fuera poco, en el Perú de aquellos años había una atmósfera antisemita soterrada y una simpatía por el fascismo y el nacionalsocialismo (Cotler, 978; Ciccarelli, 990; López Soria, 98; Molinari Morales, 2006). No ha de extrañar por ello que Blumenfeld fuera objeto de ataques y postergaciones por parte de personalidades influyentes en la Universidad de San Marcos (Alarcón, 2006).

Al finalizar la Segunda Guerra Mundial Blumenfeld frisaba los 63 años, y tenía ya diez en el Perú, país al cual había logrado traer a la parte de su familia que logró sobrevivir al nacionalsocialismo. De otro lado, Alemania (y por supuesto Dresde) se encontraba casi totalmente destruida, por lo cual un retorno quedaba excluido.

Con el paso de los años, el interés de Blumenfeld por la filosofía fue en aumento, como lo acreditan varias publicaciones aparecidas tanto en castellano como en alemán (e.g. Blumenfeld, 95, 966), alguna de ellas inclusive publicada en Kant-Studien (Blumenfeld, 96-962).

\section{Arthur Liebert}

Nacido en una familia judía, Arthur Liebert (originalmente Arthur Levy), tras unos años de desempeñarse en el mundo del comercio, estudió en la Universidad de Berlín entre 90 y 907, teniendo entre sus profesores a Wilhelm Dilthey (833-9) y también a algunos que después lo serían asimismo de Blumenfeld: Carl Stumpf, Aloys Riehl (844-924) y Georg Simmel (858-98). En 908 obtuvo el doctorado en filosofía bajo la dirección de Friedrich Paulsen (846-908) y Riehl con una tesis sobre Pico della Mirandola (463-494) (Levy, 908), pensador italiano algunas de cuyas obras había traducido y editado previamente (Liebert, 905). Desde 9, después de ser bautizado y convertirse al protestantismo, optó por el apellido Liebert (Kučinar, 205).

Hacia la finalización de sus estudios, Wilhelm Dilthey, su antiguo profesor y mentor, convocó a Liebert a colaborar en la edición de las obras de Kant a cargo de la Königlich Preussische Akademie der Wissenschaften ${ }^{1}$, siendo prontamente el secretario y asistente del gran pensador alemán.

En 90 entra a trabajar en la KantGesellschaft, la sociedad académica destinada a estudiary promover la difusión de las ideas del filósofo de Königsberg, que había sido fundada años antes por Hans Vaihinger (852-933), siendo entre 90 y 927 Stellvertretender Geschäftsführer y entre 927 y 933 su Geschäftsführer. A la par de su actividad en esa institución, Liebert enseñó psicología y pedagogía 
desde 90 en la Handels-Hochschule en la capital alemana, y tras su Habilitation, también en la Universidad de Berlín, siendo nombrado Ausserördentlicher Professor en 928, y con los derechos de un Ordinarius desde 93. En 933 fue separado de sus funciones docentes por su origen judío, a pesar de que se había convertido al cristianismo.

Liebert, tanto por su actividad al frente de la Kant-Gesellschaft cuanto por su permanente labor publicista, fue una figura muy conocida en el escenario cultural alemán de la época, así como en el extranjero, y se lo puede considerar como un excelente organizador de la actividad filosófica y el más grande difusor de la filosofía en su época.

Numerosos artículos y libros fueron el resultado de su incansable actividad (e.g. Liebert, 94, 99a, 923, 936). Das Problem der Geltung (Liebert, 94) y Wie ist kritische Philosophie überhaupt möglich - Ein Beitrag zur systematischen Phänomenologie der Philosophie (Liebert, 99b), que alcanzaron varias ediciones, fueron sus obras más influyentes en la comunidad académica, y en ellas se evidencia su postura neokantiana (Kučinar, 205; una breve valoración en castellano de su obra se encuentra en Ferrater Mora, 965).

En el año de su muerte fue publicado Der universale Humanismus (Liebert, 946), con un extenso subtítulo (Eine Philosophie über das Wesen und den Wert des Lebens und der menschlich-geschichtlichen Kultur als Philosophie der schöpferischen
Entwicklung). Se trata de una extensa obra (de la cual solo apareció el primervolumen, Grundlegung, Prinzipien und Hauptgebiete des Universalen Humanismus) que se encontraba trabajando cuando las leyes racistas de Hitler lo despojaron de su labor docente, y que solo pudo concluir durante su estancia en Inglaterra.

En este trabajo Liebert propone la unificación de los humanistas en una liga mundial (Vereinigung der Humanisten zu einem Weltbund), de la cual la sociedad Philosophia y la renovación de la KantGesellschaft serían los primeros pasos, así como la elaboración de principios para los acuerdos económicos internacionales, y sobre todo, la de un plan para la educación de los jóvenes. Los congresos y las publicaciones debían servir a estos propósitos, en tanto que un latín simplificado (ein vereinfachtes Latein) sería el idioma universal.

No solo en Alemania el nombre de Liebert circuló con frecuencia. También en América Latina, donde en calidad de director de la Kant-Gesellschaft mantuvo correspondencia con Francisco Romero (89-962), el filósofo establecido en Buenos Aires, quien junto con Alejandro Korn (860-936) había fundado una sociedad dedicada a Kant (Jalif de Bertranou, 207). Romero mantuvo al parecer una correspondencia más activa que la que tuvo Blumenfeld con él, y señala que antes de emigrar a Inglaterra en algún momento Liebert podría haber pensado en trasladarse a la Argentina (Romero, 953, 207).

Afectado por las nuevas normas raciales del gobierno nacionalsocialista Liebert 
emigró en 934 a Yugoslavia, un país que le era extraño, cuyo idioma no conocía y que además no disponía de las facilidades en materia de bibliotecas y materiales de estudio a los que estaba acostumbrado en sus años alemanes.

En Yugoslavia fue profesor en la Universidad de Belgrado y permaneció hasta 939. Los años de su estancia coincidieron con una época difícil en la vida política de Yugoslavia. Tras el asesinato en 934 del rey Alejandro Karageorgevic en Marsella (Francia), el país se inclinó progresivamente a los regímenes deAlemania e Italia, especialmente durante el gobierno de Milan Stojadinović (888-96), quien ejerció el cargo de primer ministro de 935 a 939, bajo el reinado de Pedro II. La presión política y económica del régimen nacionalsocialista sobre Yugoslavia llevó a que este país asumiera una creciente política de discriminación y vigilancia de la población judía (Ristović, 2007). Ante eso, Liebert emigró a Inglaterra (con alguna colaboración de Stefan Zweig (88-942), el gran escritor austriaco; Basta, 995), estableciéndose en Birmingham, en donde pasó por circunstancias muy difíciles e intentó sin éxito revitalizar la sociedad que fundara en Yugoslavia. Tras treceaños deemigración retornó a Alemania en 946, reincorporándose a la Universidad de Berlín, siendo elegido decano de la Facultad de Pedagogía. En ese mismo año falleció.

Poco después de haber sido separado de sus funciones docentes, Liebert fue contratado por la Universidad de Belgrado. Kajica Milanov (905-986), doctorado en la Universidad de Berlíny exalumno suyo, fue uno de los que influyó para tal contrato.
Como sucedió asimismo con Blumenfeld, tuvo que dedicar un buen tiempo y gran energía para familiarizarse con un idioma que era completamente diferente al suyo. No llegó a dominarlo, de modo tal que sus clases eran dictadas en alemán pero contaba con la ayuda de una estudiante serbia que cumplía las funciones de intérprete. Sin embargo, en una carta del 7 de mayo de 935 dirigida a Max Dessoir (867-947), su antiguo profesor, Liebert indica que la Facultad le ha solicitado que dicte algunos cursos en alemán. Su hijo sí llegóa familiarizarse con esa lengua y su ayuda le era muy útil en las actividades cotidianas que debía cumplir.

Kučinar (205) ha logrado establecer las asignaturas que enseñó Liebert durante sus años en la Universidad de Belgrado: comenzó sus actividades docentes con el curso de "Pedagogía" en 934, y continuó con temas de esta especialidad hasta el final de su estancia, intercalando asignaturas como "Filosofía contemporánea" (937) y "Grandes pensadores de la antigüedad y de los tiempos modernos" (938-939).

Establecido ya en Belgrado Liebert fundó en 936 la sociedad Philosophia y una revista del mismo nombre, entre cuyos colaboradores se encontraban Edmund Husserl (859-938), el de mayor prestigio, Karl Löwith (897-973), Martin Buber (878965), Helmuth Plessner (892-985) y Ernst Cassirer (874-945), entre otros.

El objetivo de la sociedad y de la publicación era estrictamente académico y, dado el prestigio de Liebert, contó con valiosas contribuciones de diferentes partes del mundo, destacando entre 
ellas las de algunos académicos alemanes que se encontraban también en el exilio. Importante es señalar el carácter internacional de la revista Philosophia, expresado ya en su mismo título, una palabra del latín, pero también a través de la publicación de trabajos en diferentes idiomas (e.g. Kotarbinski, 936; Meyer 936), sobre todo en los volúmenes 2 y 3.

No eran precisamente los mejores tiempos para dedicarse a actividades editoriales. Belgrado no era una ciudad con gran significación en el mundo de la filosofía, y en lo referente a la logística necesaria para editar una publicación periódica, tampoco parecía contar con todos los recursos y el personal necesarios. Agreguemos a esto que Philosophia publicaba trabajos en alemán, francés e inglés, en tanto que el idioma de uso diario en Belgrado era el serbio.

A pesar de todo esto, Arthur Liebert prosiguió adelante con su proyecto. Los años al frente de la revista Kant-Studien y de la Kant-Gesellschaft constituían un cúmulo importante de experiencia editorial así como también lo habían vinculado a muchos filósofos y estudiosos del mundo entero.

Es así que, decidido a emprender esta nueva aventura editorial, procedió a iniciar una activa e incansable campaña de difusión de su revista y de su sociedad dirigiendo cartas a numerosas personas y colegas en el mundo entero. Muchas de ellas eran también alemanas y se habían visto afectadas por las leyes racistas que las habían privada de su actividad docente o profesional, obligándolas a buscar un nuevo futuro en otras latitudes. Uno de ellas fue Walter Blumenfeld.

En su carta, con un contenido standard, Liebert informaba (e invitaba a unirse) acerca de la fundación de la sociedad "Philosophia" y de la revista del mismo nombre, que eran fundamentadas como una necesidad científica, moral y cultural (eine wissenschaftliche, als eine moralische und als eine kulturelle Notwendigkeit). ${ }^{2}$

Wirth (2005) ha efectuado una exhaustiva presentación de la incansable actividad llevada a cabo por Liebert, señalando que en diciembre de 1937, la sociedad contaba nada menos que con 635 miembros en el mundo entero, entre ellos personalidades de la cultura, la ciencia y la política. En la misma Yugoslavia el número de socios era elevado: entre otros Branislav Petronijević (1875-1954), Kajica Milanov, Jelisaveta Branković, Ernst Friedmann, H. T. Littauer, Marko Car (1859-1953), Franc Derganc (1877-1939), Albert Bazala (18771947) y Milian Schömann; un escritor y emigrante de Alemania que era asistente de Liebert y autor de varias recensiones. Un socio más, era Ivo Andrić (1892-1975), cuya novela El puente sobre el río Drina (Andrić, 2016) lo hizo mundialmente conocido y quien, años más tarde, en 1961, recibiría el Premio Nobel de Literatura.

La relación de latinoamericanos y españoles comprometidos con Philosophia es extensa. Entre los primeros se encuentran: Raimundo Lida (1908-1979, Buenos Aires, Argentina), Mariano Th. Deur (La Paz, Bolivia), Hermann Halberstaedter 
(1896-1966, Bogotá, Colombia), Leo Matthias (1893-1870, Popayán, Colombia), Guillermo Valencia (1873-1943, Popayán, Colombia), Gerhard Masur (1901-1975, Bogotá, Colombia), Baldomero Sanín Cano (1861-1957, Cali, Colombia), Antonio Sánchez de Bustamantey Montoro (19101984, La Habana, Cuba), Paul Honigsheim (1885-1963, Panamá), Octavio Méndez Pereira (1887-1954, Panamá), Richard Behrendt (1908-1972, Panamá), Walter Blumenfeld (Lima, Perú) y Francisco Aguilar (1887-?, Lima, Perú). En España se contaban los siguientes miembros: Ángel Rodríguez-Bachiller (1901-1983, Valladolid), Jaime Serra-Hunter (18781943, Barcelona), Luis Recasens Siches (1903-1977, Madrid) y Joaquín Xirau (18951946, Barcelona).

La sociedad también tuvo entre sus integrantes, aparte de Blumenfeld, a otros importantes psicólogos europeos, como David Katz (1884-1953), Wolfgang Köhler (1887-1967), Kurt Goldstein (1878-1965), William Stern (1871-1938), Kurt Lewin (1890-1947) y Karl Bühler (1879-1963), entre otros.

El prestigio de Liebert motivó que Branislav Petronijević, uno de los más destacados filósofos locales y exsecretario (1932-1933) de la Real Academia Serbia, lo propusiera como miembro de ella. Lamentablemente, las presiones del gobierno alemán hicieron que Liebert perdiera su posición docente en la Universidad de Belgrado y que la proposición de incorporarlo a la Academia no prosperará (Kučinar, 2015). Asimismo, la fundación de la sociedad Philosophia constituyó un importante estímulo para la creación de la Sociedad Serbia de Filosofía en 1938.

\section{Lima y Belgrado}

¿Se conocían Blumenfeld y Liebert? Es probable que sí: Liebert se había ganado un nombre al frente de la Kant Gesellschaft, que alcanzó durante sus años de administración una gran cantidad de nuevos socios y recobró la fuerza y presencia intelectual que había perdido tras la Primera Guerra Mundial. La revista Kant-Studien, a su vez, tenía asimismo un gran prestigio académico (que por cierto conserva hasta hoy). ${ }^{3}$ Además, como ya se dijo, Liebert se desempeñaba como profesor en la Universidad de Berlín y había dado a la luz numerosas publicaciones en el campo de la filosofía, la ética y en la literatura.

Blumenfeld, por su parte, mostraba asimismo un gran interés por temas filosóficos. Muchos de sus profesores en la Universidad de Berlín, como lo hemos señalado, eran destacados filósofos, y él mismo había incursionado en sus publicaciones en tópicos propios de la filosofía y la epistemología (Blumenfeld, 1920, 1925b). En sus años postreros ese interés se tornaría aún más notorio. Se puede deducir, por ello que ambos se conocían ya en Alemania. Abona a favor de esta suposición el trato cercano que caracteriza la comunicación epistolar entre ambos: El "lieber Freund" (querido amigo) es la forma en que se dirige el uno al otro, diferente del habitual y formal, "sehr geehrter Herr Professor" (muy estimado señor profesor). 
Las cartas conservadas entre Liebert y Blumenfeld corresponden a un corto periodo (1936-1939), o sea en realidad poco tiempo después de que se establecieran en sus nuevos destinos, y están sobre todo referidas a la sociedad Philosophia y su revista, pero informan de modo tangencial acerca de la situación de ellos y sobre sus nuevos entornos culturales. La tabla 1 presenta las cartas cursadas entre ambos.

Tabla 1. Correspondencia entre Walter Blumenfeld y Arthur Liebert

\begin{tabular}{|lll|}
\hline Año & De W. Blumenfeld a A. Liebert & De A. Liebert a W. Blumenfeld \\
\hline 1936 & 15 de noviembre & 15 de diciembre \\
& 8 de febrero & 26 de marzo \\
1937 & 20 de febrero & 27 de setiembre \\
& 1 de octubre & 1 de octubre \\
& 27 de noviembre \\
& 4 de enero & 28 de marzo \\
1938 & 22 de febrero marzo & 18 de abril \\
& 14 de mayo & 24 de mayo \\
& & 21 de marzo \\
1939 & 25 de mayo & 24 de junio \\
\hline
\end{tabular}

Nota:Cartas conservadasenel ArchivoArthur Liebert en posesión del Prof. Dr.Zdravko Kučinar.

La correspondencia se inicia con una carta de Blumenfeld (1936) a Liebert fechada el 15 de noviembre de 1936. Después de alegrarse por la posibilidad de leer la revista que planea Liebert, comenta en los siguientes términos la posibilidad de recensionar en el Perú algún libro de su colega:

Me agradaría mucho declararme dispuesto a recensionar algunas de sus obras, si es que aquí existiera alguna revista. No sé cómo es el estado de cosas en Belgrado, pero aquí prácticamente no hay alguna revista de significado, así como alguna persona que compre libros, mucho menos los estudiantes. Los profesores deben preparar "copias" de sus clases, las cuales son cuidadosamente memorizadas pero rara vez comprendidas. Voilà tout. Ellas constituyen al mismo tiempo el remplazo de la asistencia a clases.

Liebert, en carta del 27 de setiembre de 1937, le expresa a Blumenfeld su proyecto de lanzar una edición en español de Philosophia y que Lima puede ser el centro para lanzar esa edición, considerado que Blumenfeld se encuentra allí. Le pide información acerca de cómo están las imprentas en el Perú. Tiempo después, en carta del 4 de enero de 1938 , volviendo al tema de la posibilidad de conseguir miembros para la sociedad de Liebert y suscriptores para su revista, Blumenfeld se muestra pesimista acerca de ambas cosas:

Soy más escéptico acerca de una propaganda en español, al menos en el Perú. Pues aquí hay poco espíritu emprendedor 
y un escaso casi inexistente interés por los libros; tampoco existe ningún establecimiento en el que se pueda confiar y el distribuidor es totalmente incapaz de hacer tales cosas. Piense Usted que toda Sudamérica puede tener más de 60 millones de habitantes (no hay cifras precisas debido a la ausencia de censos) y de estos probablemente 40 o 50 millones son analfabetos en el sentido estricto del término.

En las postrimerías de 1936, el 15 de diciembre, Liebert escribe a su corresponsal en Lima informándole que ha recibido su carta del 15 de noviembre así como un ejemplar de su libro Sinn und Unsinn (Blumenfeld, 1933), indicándole que aunque el libro es relativamente antiguo, tiene la intención de comentarlo en The Philosophical Review, revista norteamericana en la cual él forma parte del Comité Editorial. Le informa asimismo que solo espera el trabajo de Husserl para lanzar el primer número de Philosophia:

Si el buen Edmund Husserl no demorara tanto con sus correcciones, ya habría aparecido el primer volumen. Tengo la esperanza de que eso suceda a comienzos del próximo año.

En carta del 1 de octubre de 1937 Liebert le escribe a su corresponsal en Lima indicándole que hay alguien interesado en esa ciudad en la sociedad y en la revista, y le señala que si bien ha logrado adentrarse en los misterios del idioma serbio no posee las habilidades suficientes en el manejo del castellano como para responder al interesado, motivo por el cual le remite la carta enviada desde Lima y le pide que se comunique con esa persona para mayores detalles, al mismo tiempo que le informa del envío de dos ejemplares de Philosophia, uno de los cuales está destinado al solicitante de información.

Ya en una carta previa le había informado de los cautelosos avances que hacía en el conocimiento del serbio señalando que lo hablaba como un esquimal hablaría italiano. El 18 de abril de 1938, al comunicarle que tiene en su poder una recensión de su libro Jugend als Konfliktsituation, Liebert informa a Blumenfeld que viaja con cierta frecuencia a Laubach, y que su hijo ya no está con él sino que se ha mudado a la ciudad de Novi Sad.

Poco tiempo después, en una nueva carta fechada el 24 de mayo de 1938 Liebert, informa a Blumenfeld de las dificultades económicas que enfrenta para poder sacar cada nueva edición de Philosophia, dado que tiene que asumir todos los costos y esperar que hayan sido plenamente cubiertos para lanzarse a la edición del siguiente volumen, además de encargarse hasta de los menores detalles de la edición. Esta situación, señala, jamás la había tenido cuando estaba al frente de la Kant-Gesellschaft y de Kant-Studien:

Recibo una gran cantidad de manuscritos, pero no he podido conseguir un apoyo estatal o de ningún tipo para Philosophia, a pesar de haberme dedicado mucho a buscarlo. También en los Estados Unidos he movilizado a mis contactos y llegado a las más diversas instituciones. Debo encargarme de cada edición a través de un trabajo muy 
minucioso. Cuanto me agradaría lanzar cada nuevo número en una secuencia más rápida. Lamentablemente no puedo porque debo pagar completamente la edición de cada número para poder dar a la prensa el siguiente. El último número (2) ha costado alrededor de 50 mil dinares. Hasta ahora se ha pagado $36 \mathrm{mil}$. He calculado que podré imprimir el siguiente número en junio o julio como la fecha más temprana, el cual recién aparecerá teniendo en cuenta las circunstancias técnicas tan particulares acá en el otoño.

En efecto, muchos de los manuscritos remitidos a la redacción de Philosophia estaban redactados a mano, motivo por el cual el propio Liebert tenía que encargarse de mecanografiarlos y prepararlos para su envío a la imprenta. En esto contaba con la valiosa ayuda de un asistente de origen alemán. Asimismo, lo afirmado en la primera línea («recibo gran cantidad de manuscritos») queda evidenciado en el aumento del número de páginas con cada nueva entrega: 442 en el primer volumen (1936), 451 en el segundo (1937) y 621 en tercero y último (1938) (Kropp, 1947).

El 21 de marzo de 1939, o sea pocos meses antes de que estallara la Segunda Guerra Mundial, Liebert escribe a Blumenfeld señalándole que se encuentra bien de salud y que está a la espera de un cambio sustancial en su situación como profesor en la Universidad y con respecto a su revista, en un tono positivo. Señala sin embargo que después de muchos trajines («nach Überwindung unbeschreiblicher und unvorstellbarer Schwierigkeiten») ha logrado la edición del volumen de
Philosophia, en el cual aparece un trabajo de Blumenfeld (1938d) y que le enviará prontamente la revista así como cincuenta separatas. Hace referencia asimismo a lo que su corresponsal desde Lima le informa sobre la situación en el Perú, esto es que las condiciones impuestas por el gobierno peruano para la inmigración se han vuelto más complejas y difíciles. El mismo señala que no hay día en que no reciba malas noticias de Alemania sobre el particular.

En efecto, Blumenfeld, el 18 de febrero de 1938, le escribe a Liebert lo siguiente:

El ánimo en general aquí en contra de los extranjeros y de los judíos en particular ha empeorado debido a una intensa propaganda, y la inmigración está prácticamente cerrada. La única excepción consiste en que los hijos pueden pedir a sus padres si tienen por encima de los 60 años, y si ellos mismos tienen más de dos años en el país. Contratos de trabajo en principio no están permitidos.

Los cuidados que Liebert prodigaba a cada nueva edición de Philosophia permitieron que los números de ellos estuvieran casi libres de errores. Blumenfeld felicita en una de sus cartas, del 25 de mayo de 1939, a Liebert después de haber recibido el segundo volumen de la revista en el cual aparece su trabajo (Blumenfeld, 1938) por el hecho de que la edición es muy pulcra, algo que él no puede decir de muchos de los trabajos que él ha publicado inclusive en castellano en Lima.

"Der Begriff des Ursprungs in Metaphysik und Wissenschaft" es un trabajo denso 
en el cual Blumenfeld relieva la complejidad del concepto de Urspung (origen), que determina múltiples y muchas veces antagónicos sentidos en el mundo de la metafísica, razón por la cual considera que la explicación del mismo debe más bien orientarse de acuerdo con la lógica. Este artículo (bastante extenso, por lo demás) es una clara demostración de los intereses filosóficos que el psicólogo alemán siempre tuvo. ${ }^{4}$

Como hemos señalado, Blumenfeld estaba encargado de promover la difusión de Philosophia en el Perú. El 8 de febrero de 1937 Blumenfeld le escribe a Liebert que ha cumplido con sus tareas. Menciona a dos personas que podrían formar parte del comité directivo de la revista, refiriéndose a Honorio Delgado (1892-1969) y Mariano Iberico (1892-1974), pero señala que el primero se encuentra de viaje hasta fines de ese mes, de modo tal que no le ha sido posible conversar con él. Con referencia al segundo, manifiesta que él había recibido la carta de Liebert y que pronto le contestaría dado que se encontraba muy ocupado en ese momento. Pero agrega, «ojalá que cumpla con su palabra, pues en este asunto no se pueden esperar estándares europeos». Indica asimismo que la posición de ambos académicos peruanos no influiría en mucho excepto en que por medio de ellos le habría sido posible acceder a la muy delgada capa intelectual en el Perú.

No le faltaba razón a Blumenfeld con respecto a Delgado e Iberico. En los años '3o, cuando él arriba al Perú, ambos eran dos de los intelectuales más reconocidos en el escenario cultural del país andino. Delgado, médico especializado en psiquiatría, había introducido el psicoanálisis en el mundo de habla hispana y, aparte de visitar a Sigmund Freud en las oportunidades en que viajó a Europa, mantuvo una correspondencia con él. Iberico, por su parte, era un abogado de profesión pero con una marcada vocación por la filosofía, llegando a ser el filósofo peruano más importante en el siglo XX. Ambos eran amigos y habían escrito en conjunto un libro de psicología (Delgado \& Iberico, 1933), que ejercería gran influencia en los estudios de esa especialidad en el Perú. En una carta posterior, del 25 de mayo de 1939, le informa que hay dos personas más en el Perú interesadas en suscribirse a Philosophia: Francisco Miró Quesada (1918-2019) y Eugenio Alarco (1908-?), quienes con el paso de los años harían una brillante carrera filosófica.

Blumenfeld por su parte solicita a Liebert que el artículo que le ha remitido sea publicado prontamente indicando que la única carta de presentación que tienen los exiliados es su trabajo y sus publicaciones mediante las cuales pueden demostrar su capacidad de trabajo. El 24 de junio de 1939 Liebert (1939b) le escribe a Blumenfeld:

Se me ha hecho imposible continuar con mi existencia y mi trabajo en Belgrado; mi hijo y yo estamos bajo vigilancia policial, a pesar de que la Universidad hace solo dos meses me ha renovado el contrato por tres años más y haber sido confirmado por el senado de ella. Pero Inglaterra ha intervenido en mi ayuda de modo enérgico (....) 
Tengo todo listo para mi viaje, he vendido lo que tenía en casa y mis libros y solo tengo que esperar 14 días pues mi hijo está desde hace nueve semanas enfermo, felizmente se está recuperando pero aún no está en condición de emprender viaje.

\section{Philosophia}

Como se habrá visto, la comunicación epistolar entre Blumenfeld y Liebert tiene como eje central a la sociedad Philosophia y a la revista del mismo nombre. ¿Qué animó a Liebert, en tiempos tan inciertos, a crear una sociedad académica como Philosophia? Debemos suponer que con ella buscaba que continuar con las actividades que más conocía, esto es con la conducción exitosa de la Kant Gesellschaft y de la revista Kant-Studien. Como hemos señalado, tanto esa sociedad como la revista tuvieran una edad de oro durante su gestión, y ello más las experiencias acumuladas fueron importantes para que él tomara la iniciativa de fundar Philosophia. Kučinar (2015) señala que fueron creadas como una suerte de sustitutos de la sociedad y revista alemanas dedicadas a Kant, que habían caído en manos de conductores de orientación nacionalsocialista. Algunos de los convocados a apoyar y participar en la nueva sociedad y en su revista así lo reconocieron. Halberstaedter, por ejemplo, como lo señala en carta que dirige a Liebert el 21 de noviembre de 1936.

El paso del tiempo nos permite afirmar que Liebert subestimó las dificultades que habría de enfrentar en Belgrado tanto para dirigir la sociedad pero sobre todo para la publicación de la revista. Líneas arriba hemos tratado sobre el particular. Y, sin embargo, Liebert continuó adelante con su proyecto. En las cartas que cruza con Blumenfeld informa acerca de la sociedad, de sus miembros latinoamericanos, solicita la intervención de su corresponsal en Lima a la búsqueda de nuevos socios, y le hace recordar el pago de la membresía.

Philosophia, la revista, apareció en 1936 y tenía como subtítulo Philosophorum nostri tempori vox universa. El primer número la hizo famosa dado que incluye el trabajo ya mencionado de Husserl (1936), en realidad el último trabajo de quien era en su momento el más importante e influyente filósofo de su época. La contribución de Husserl amplía notoriamente las ideas que había presentado en una conferencia suya en el Círculo Filosófico de Praga cuyo nombre oficial en francés era Cercle philosophique de Prague pour les recherches sur l'entendement humain (Checoslovaquia), a la cual asistieron, entre muchos otros, Felix Kaufmann (1895-1949), Hans Lessner, Jan Patócka (1907-1977), Ludwig Landgrebe (19021991) y Alfred Schütz (1899-1959) (RizoPatrón de Lerner, 2015).

La experiencia de Liebert en materia de edición de revistas hizo que retrasara en medio año la publicación del primer número de Philosophia a la espera de la contribución de Husserl, cuyo nombre sería un magneto para los lectores, como en efecto lo fue. Aparte del prestigio de incluir el trabajo de Husserl en Philosophia, Liebert estaba interesado en el tema abordado por el gran filósofo, 
pues él mismo había tratado el tema de la crisis en varias de sus publicaciones (Liebert, 1923, 1936b).

La revista tuvo una existencia limitada a los años de Liebert en Belgrado. Pero su corta existencia no significa que no haya tenido importancia. Se trata, al parecer de la más importante publicación periódica en el exilio alemán (Mehring, 2018). Hoy es recordada fundamentalmente por el trabajo de Husserl.

\section{Conclusiones}

La irrupción del nazismo en Alemania y su bárbara intromisión en la vida universitaria de esa nación fue la causa de la diáspora de intelectuales, investigadores, estudiosos y artistas alemanes que, ya porque eran judíos o se oponían al régimen de Hitler, fueron separados de sus funciones. No hubo lugar en el que no terminara algún profesor alemán. Así, por ejemplo, el medievalista Erich Kantorowicz (1895-1963) y el crítico literario Erich Auerbach (1892-1957) (que alcanzarían posteriormente fama mundial) pasaron algunos años nada menos que en Turquía antes de trasladarse a los Estados Unidos. Turquía estaba lejos de Alemania, pero en realidad se encontraba todavía en los límites de lo imaginable en materia de lugares adonde emigrar. Karl Löwith, después de un tiempo en Italia, emigró al Japón, en donde permaneció hasta que este país se alió a Alemania, razón por la cual terminó trasladándose a los Estados Unidos. Como puede verse, los casos de Blumenfeld y de Liebert en modo alguno no fueron los únicos.
Las cartas que sirven de base para este estudio nos permiten tener una imagen de los problemas que ambos, cada uno en su respectiva locación, experimentaban. Uno de ellos, tal vez el más inmediato, era el del conocimiento del idioma. Sin un manejo relativamente bueno del idioma es imposible adaptarse a cualquier realidad. Blumenfeld emprendió el trabajo de aprender el castellano y logró dominarlo a un modo tal que terminó publicando importantes estudios y libros en ese idioma.

Lo segundo es el problema de las facilidades para la investigación a las que ambos estaban acostumbrados en Alemania y que estaban ausentes en sus nuevos lugares de residencia, especialmente en el caso de Blumenfeld en el Perú. Es de imaginarse las dificultades que ambos tuvieron que afrontar para continuar con sus trabajos. La ausencia de adecuadas bibliotecas y hemerotecas es algo que más debe haber afectado a ambos, pero tampoco constituye un caso excepcional. Auerbach escribió su famoso libro Mimesis (Auerbach, 1946) en Turquía, sin contar con los textos de consulta que él estaba acostumbrado a frecuentar en Alemania (Bormuth, 2020).

Pero una cosa debe destacarse y esa es la profunda convicción que animaba a Liebert a continuar con proyectos académicos que parecían casi inviables en Yugoslavia y en los tiempos que se vivían. Recurriendo a sus extensos contactos casi en el mundo entero despliega una actividad que bien merece el calificativo de incansable para ganar nuevos socios para Philosophia y difundir la revista que 
editaba, que era remitida desde Belgrado a todos sus suscriptores.

Lima y Belgrado generan en Blumenfeld y Liebert apreciaciones diferentes. Para el primero la capital peruana es una ciudad muypocoestimulanteen el plano intelectual yacadémico. Blumenfeld (1938b) lo señalaen una de sus cartas, fechada el 14 de mayo de 1938, al escribir que se encuentra en un lugar al margen de la cultura. Solicitandoa Liebert quepubliqueprontamentesu trabajo, ledice:

Para personas que como yo estamos al margen de la cultura, la situación es mucho más difícil que para aquellos colegas que viven en Europa o en los Estados Unidos, porque no tenemos ninguna posibilidad del contacto personal a través de viajes o congresos. (el subrayado es nuestro, N. de los A.)

Liebert no es tan duro en sus expresiones acerca de Belgrado, aunque se refiere reiteradamentea los problemas queexperimenta para continuar con sus emprendimientos académicos.

Aparte de las dificultades para editar Philosophia, en otros planos Liebert es mucho menos crítico con respecto a lo que vivía en Belgrado. En la ya mencionada carta a Max Dessoir le manifiesta estar satisfecho en líneas generales con la universidad, reconociendo que tiene algunas limitaciones debido a su juventud, pero que los estudiantes son excelentes. De excelentes asimismo califica sus relaciones con los colegas de esa casa de estudios, con algunos de los cuales - afirma - mantiene estrecho contacto. Con respecto a Belgrado, le hace saber que muchas calles del antiguo barrio turco están siendo arrasadas para dar lugara modernos edificios, agregando que la cercanía deyacimientos de mármol permite el empleo de este costoso material en las construcciones. Algo semejante informa en otra carta, del 17 de abril de 1935, dirigida esta vez a Ernst Tiessen, el rector de la Handels-Hochschule en Berlín, en la que agrega que el país tiene zonas particularmente bellas, especialmente en el sur (Macedonia), aunque anota asimismo que "debemos vivir muy modestamente pues los ingresos de los profesores universitarios son muy limitados" (wir müssen hier ganz bescheiden leben, die Einnahmen auch der Universitätsprofessoren sind ganz gering).

Por otro lado, la recepción de Liebert en Yugoslavia fue favorable, tantoasí queél integró la delegación yugoslava al VIII Congreso Internacional de Filosofía que se celebró en Praga en 1934.

Como hemos dicho al comenzar esta comunicación, las diferencias entre ambas ciudades son grandes y lo eran también en el pasado. Lima, que en la época de la dominación española, era la gran metrópoli de la región sudamericana, perdió en el siglo XIX mucho de esa significación, y ubicada al otro lado de los Andes, ella (y el Perú también) atrajo poca emigración europea, en todo caso mucho menor que la que se estableció en ciudades como Buenos Aires, Montevideo o Río de Janeiro. Las turbulencias políticas del país y su escaso nivel de desarrollo social determinaron una atmósfera cultural muy limitada, como también limitado era progreso en 
el plano científico. A eso hace referencia Blumenfeld en sus cartas.

Aunque Belgrado no era una ciudad con un nivel de desarrollo cultural y científico comparable con urbes como Berlín, París o Londres, había experimentado un gran crecimiento (Lenger, 2014); y su relativa cercanía a las grandes urbes y centros científicos europeos hacía deella un lugar mucho más interesante que la capital peruana para muchos emigrantes, que además, tenían la esperanza de un pronto derrumbe del régimen. Eso explica, por ejemplo, que algunas personalidades dela ciencia alemana se trasladaran a ese país para continuar su trabajo, al menos por un tiempo.

El caso más conocido es el de Ferdinand Blumenthal (1870-1941), prestigioso especialista en cáncer, directordel instituto de investigación de esta enfermedad en el mundialmente conocido hospital Charité, de Berlín, yeditor de la Zeitschrift für Krebsforschung, quien debido a su condición de judío, fue separadode todos sus cargos. Tras su forzado retiro, se trasladó a Suiza, y poco después aceptóla invitación que le hiciera la Facultad de Medicina de la Universidad de Belgrado para asumir funciones docentes en ella, que desempeñaría hasta 1937, viéndose obligado a emigrar nuevamente cuando el gobierno alemán ejerció presiones paraqueselodespidiera (Milosavljević, 2010).

Pero lo interesante acá es destacar la presencia de este importante científico en Yugoslavia y las posibilidades que tuvo de continuar en alguna medida con su trabajo, en parte también debido a su cercanía a los centros científicos europeos.
Blumenfeld, con solo poco tiempo todavía en el Perú y con casi nula producción escrita en castellano se preocupa sobre todo porquesus libros publicadosenalemán (Sinn und Unsinn y Jugend als Konfliktsituation; Blumenfeld, 1933, 1936) sean lo más difundidos a través de recensiones y comentarios, algo que Liebert intenta por todos los medios a sualcance. Inclusive habla dela posibilidad de publicar una recensión en alguna revista norteamericana.

En tanto que Blumenfeld permanecería el resto de su vida en el Perú, Liebert como hemos señalado emigró a Inglaterra. En el Perú Blumenfeld vería peligrar su situación cuando por intervención precisamente de Honorio Delgado y con el apoyo de Mariano Iberico Rodríguez, el Instituto de Psicología y Psicotécnica es cerrado e inclusive su condición de profesor en la Facultad de Letras corre peligro. En el marco de esa situación busca posibilidades de emigrar como lo demuestra su correspondencia con otros intelectuales latinoamericanos. A partir de los años 40 sin embargo su situación se estabiliza en el Perú y el resto de su vida se quedaría en la nación andina. Delgado e Iberico serían considerados por él, en el futuro, como sus enemigos (León, 2016).

Philosophia publicó un trabajo suyo relativamente extenso, el que sin embargo no alcanzó mayor difusión en el Perú dado que estaba redactado en alemán. Asimismo apareció una recensión de su libro Sinn und Unsinn. Esta última obra, publicada en alemán en 1933, sería vertida al castellano muchos años después (Blumenfeld, 1949) y dada a la luz en la 
editorial bonaerense Losada, que dirigía Francisco Romero.

Liebert por su parte fracasa en el intento de incorporarse a la academia de ciencias yugoeslava y en 1939 siente que corre peligro en Yugoeslavia, razón por la cual decide emigrar nuevamente, esta vez a Inglaterra, estableciéndose en Birmingham en donde vive en condiciones sumamente difíciles. Terminada la guerra retorna en 1946 a Alemania y se desempeña como profesor en la Universidad de Berlín, ocupando el decanato de la Facultad de Educación, para poco tiempo después fallecer.

Unas últimas líneas acerca de la personalidad de cada uno de ellos. La permanente y rasante actividad de Liebert nos lo muestra como una persona con una inconmovible convicción de la importancia de la filosofía en la vida social y poseída por un acendrado sentido ético y una vocación humanista. A lo largo de toda su vida, esa convicción estuvo reforzada por lo que podríamos llamar grosso modo una gran capacidad comunicativa y a una sociabilidad ágil que lo llevó a relacionarse, por medio de su trabajo en las sociedades que dirigió, las revistas que editó, las clases en las universidades en las que enseñó y la innumerable cantidad de conferencias que dictó, con numerosos intelectuales a los que supo ganar para su causa.

En el caso de Blumenfeld el compromiso con la filosofía y con la ética era asimismo intenso, pero creemos, su temperamento más bien introvertido y su interés por temas sumamente teóricos (como aquel al que dedicó el trabajo que publicara en Philosophia) no lo motivaban mucho a un contacto social o a una actividad tan intensamente comprometida con la difusión de sus puntos de vista y planteamientos como sucedió con Liebert. Anotemos, por último, lo que el propio Blumenfeld señala, al indicar que Lima no era un lugar en el cual el interés por la vida intelectual estuviera muy difundido, algo que sí sucedía en Belgrado.

En la capital peruana no existió, al menos durante todos los años que Blumenfeld vivió en ella, ninguna casa editorial importante que pudiera publicar obras como las de él u otros estudiosos, así como tampoco existían revistas especializadas en temas de psicología y filosofía. La Academia Nacional de Ciencias del Perú y otras sociedades científicas tenían escasa repercusión en la sociedad y su existencia se limitaba a ocasionales reuniones.

\section{Financiamiento}

La presente investigación fue autofinanciada.

\section{Conflictos de interés}

Los autores declaran que no tiene conflictos de interés. 


\section{Referencias}

Alarcón, R. (200o). Historia de la psicología en el Perú. De la Colonia a la República. Universidad Ricardo Palma.

Alarcón, R. (2006). La contribución de Walter Blumenfeld al desarrollo de la psicología en Perú. Revista de Historia de la Psicología, 27(1), 79-93.

Andrić, I. (2016). Un puente sobre el Drina. Debate (original: Na Drini ćuprija, 1945).

Auerbach, E. (1946). Mimesis: Dargestellte Wirklichkeit in der abendändischen Literatur. Francke.

Basta, D. N. (1995). Časopis Philosophia i njegov osnivač Artur Libert. Humboltov Klub Srbija Glasnik, 1, 7-12.

Benzenhöfer, U., \& Hack-Molitor, G. (2017). Zur Emigration des Neurologen Kurt Goldstein. Klemm \& Oelschläger.

Bettauer. H. (1922). Die Stadt ohne Juden. Ein Roman von übermorgen. Gloriette Verlag.

Böhne, E., \& Motzkau-Valeton, W., (Eds.) (1992). Die Künste und die Wissenschaften im Exil 1933-1945. Lambert Schneider.

Bormuth, M. (2020). Erich Auerbach - Kulturphilosoph im Exil. Wallstein.

Blumenfeld, W. (1913). Experimentelle Untersuchungen über die scheinbare Grösse im Sehraume. Zeitschrift für Psychologie, 65, 241-404.

Blumenfeld, W. (1920). Zur kritischen Grundlegung der Psychologie. Reuther und Reichard.

Blumenfeld, W. (1923). Untersuchungen über die Formvisualität. Zeitschrift für Psychologie, 91, 1-82 y 236-292.

Blumenfeld, W. (1925a). Das Suchen von Zahlen im begrenzten ebenen Felde und das Problem der Abstraktion. Zeitschrift für angewandte Psychologie, 26, 58-107.

Blumenfeld, W. (1925b). Historische Wissenschaft und Psychologie. Jahrbuch für Philologie, 1, 269-299. 
Blumenfeld, W. (1931a) Über quantitative und qualitative Bewertung von Testleistungen. Zeitschrift für angewandte Psychologie, 40, 209-230.

Blumenfeld, W. (1931b). Urteil und Beurteilung. Archiv für de gesamte Psychologie, Ergänzungsband, 3, 1-623.

Blumenfeld, W. (1933). Sinn und Unsinn. Reinhardt.

Blumenfeld, W. (1936). Jugend als Konfliktsituation. Philo Verlag.

Blumenfeld, W. (1938). Der Begriff des Ursprungs in Metaphysik und Wissenschaft. Philosophia, 3, 220-251.

Blumenfeld, W. (1946). Introducción a la psicología experimental. Antártica.

Blumenfeld, W. (1949). Sentido y sinsentido. Losada.

Blumenfeld, W. (1951). La antropología filosófica de Martin Buber y la filosofía antropológica. Un ensayo. Universidad Nacional Mayor de San Marcos - Sociedad Peruana de Filosofìa.

Blumenfeld, W. (1961-1962). Die Grundlagen der Ethik Nicolai Hartmanns. KantStudien, 53, 3-28.

Blumenfeld, W. (1966). Contribuciones críticas y constructivas a la problemática de la ética. Universidad Nacional Mayor de San Marcos.

Ciccarelli, O. (1990). Fascism and politics in Peru during the Benavides regime, 1933-1939: the Italian perspective. Hispanic American Historical Review, 70(3), 405-432.

Cotler, J. (1978). Clases, estado y nación en el Perú. Instituto de Estudios Peruanos.

Delgado, H., \& Iberico, M. (1933). Psicología.

Fermi, L. (1971). Inmigrantes ilustres. La historia de la migración intelectual europea 1930-1941. Bibliográfica Omeba.

Ferrater Mora, J. (1965). Diccionario de filosofía. Sudamericana (vol. 2., entrada "Liebert, Arthur, 1878-1946, 58-59. 
Feuchtwanger, L. (1933). Die Geschwister Oppermann. Querido.

Fleck, Ch. (2015). Etablierung in der Fremde. Vertriebene Wissenschaftler in den USA nach 1933. Campus.

Heilbut, A. (1983). Exiled in Paradise, German refugee artists and intellectuals in America from the 1930 s to the present. Beacon Press.

Husserl, E. (1936). Die Krisis der europäischen Wissenschaften und die traszendentale Phänomenologie. Philosophia, 1, 77-176.

Jalif de Bertranou, C. A. (2017). Francisco Romero acerca de Kant y los orígenes del idealismo alemán. Conferencia inédita de 1931. Cuyo. Anuario de Filosofia Argentina y Americana, 34, 199-223.

Kellerhoff, S. F. (2015). Mi lucha: la historia del libro que marcó el siglo XX. Crítica.

Kotarbinski, T. (1936). Les idées fondamentales de la théorie générale de la lutte. Philosophia, 1, 196-214.

Kropp, G. (1947). “Philosophia”, herausgegeben von Arthur Liebert, in der Emigration. Zeitschrift für Philosophische Forschung, 2-3, 403-411.

Kučinar, Z. (2015). Arthur Liebert, Život i dela/Leben und Werke/ Life and Works. Dosije Studio.

Leff, L. (2019). Well worth saving. American universities' life-and-death decisions on refugees from Nazi Europa. Yale University Press.

Le Rider, J. (2016). Los judíos vieneses en la Belle Époque. Ediciones del Subsuelo.

León, R. (1983). Un pionero de la psicología en América Latina: Walter Blumenfeld. Revista Latinoamericana de Psicología, 15, 433-452.

León, R. (Ed.) (2016). Cartas desde el exilio. La correspondencia entre Walter Blumenfeld y Rodolfo Mondolfo (1948-1966). Universidad Ricardo Palma.

Lenger, F. (2014). Metropolen der Moderne. Eine europäische Stadtgeschichte seit 1850. Beck. 
Levy, A. (1905). Die Philosophie Giovanni Pico della Mirandola. Ein Beitrag zur Philosophie der Frührenaissance. Inaugural-Dissertation zur Erlangung der Doktorwürde genehmigt von der Philosophischen Fakultät der Friedrich-WilhelmsUniversität zu Berlin.

Liebert, A. (Ed.) (1905). Giovanni Pico della Mirandola. Ausgewählte Schriften. Eugen Diderichs.

Liebert, A. (1914). Das Problem der Geltung. Reuther \& Reichard

Liebert, A. (1919a). Vom Geist der Revolution. Collignon.

Liebert, A. (1919b). Wie ist kritische Philosophie überhaupt möglich? Ein Beitrag zur systematischen Phänomenologie der Philosophie. Felix Meiner.

Liebert, A. (1923). Die geistige Krisis der Gegenwart. Pan-Verlag R. Heise.

Liebert, A. (1936). Die Krisis des Idealismus. Rascher.

Liebert, A. (1946). Der universale Humanismus. Eine Philosophie über das Wesen und den Wert des Lebens und der menschlich-geschichtlichen Kultur als Philosophie der schöpferischen Entwicklung. Rascher.

López Soria, I. (1981). El pensamiento fascista. Mosca Azul.

Mehring, R. (2018). Philosophie im Exil: Emil Utitz, Arthur Liebert und die Exilzeitschrift Philosophia: Dokumentation und Schicksal zweier Holocaust-Opfer. Königshausen \& Neumann.

Meyer, H. (1936). Ranke the historian - a ninetheeth century thinker. Philosophia, $1,220-242$.

Milanov, K. (1938). Recension de Sinn und Unsinn, de Walter Blumenfeld (1933). Philosophia, 3, 578-581.

Milosavljević, O. (2010). Savremenici fašizma, knjiga 2, Jugoslavija u okruženju, 19331941. Helsinški odbor za ljudska prava u Srbiji

Molinari Morales, T. (2006). El fascismo en el Perú. La Unión Revolucionaria 19311936. Universidad Nacional Mayor de San Marcos. 
Reichgestztblatt (1933). Gesetz zur Wiederherstellung des Beamtemtums, 7 de abril, I. $175-177$.

Ristović, M. (2007). “Unsere” und “fremde” Juden: Zum Problem der jüdischen Fluchtlinge in Jugoslawien 1938-1941. En: A. Hilbrenner \& D. Dahlmann (Eds.), Zwischen grossen Erwartungen und bösem Erwachen. Juden, Politik und Antisemitismus in Ost- und Südosteruopa 1918-1945, (pp. 191-215). Ferdinand Schöningh.

Rizo-Patrón de Lerner, R. (2015). El exilio del sujeto: mitos modernos y posmodernos. Pontificia Universidad Católica del Perú.

Romero, F. (1953). Estudio de historia de las ideas. Losada,

Romero, F. (2017). Epistolario (Selección). Corregidor (edición y notas de Clara Alicia Jalif de Bertranou).

Strauss, H. A., \& Röder, W. (Eds.) (1983). International Biographical Dictionary of Central European Emigrés 1933-1945. Saur.

Wirth, G. (2005). Auf dem “Turnierplatz” der geistigen Auseinandersetzungen. Arthur Liebert und die Kantgesellschaft (1918-1948/1949). Ludwigsfelder Verlagshaus. 


\section{Notas}

1 Dilthey, integrante de la Akademie, había propuesto en 1894 y logrado que esta institución emprendiera la edición de los Kants Gesammelte Schriften, que comenzaron a aparecer desde 1902 en sus diferentes secciones: Obras (Werke, volúmenes 1 al 9), Correspondencia (Briefwechsel, 10-13), Legado manuscrito (Handschriftliche Nachlass, 14-23) y Lecciones (Vorlesungen, 24-29). El responsable general de tan monumental trabajo fue Dilthey, que tuvo a su cargo asimismo la edición de los volúmenes 1 al 9. Rudolf Reicke (1825-1905) editó hasta su fallecimiento los volúmenes 10 al 13, y después de él continuaron el trabajo Rose Berger, Johannes Reicke y Paul Menzer. Los manuscritos, que fueron presentados en los volúmenes del 14 al 23, fueron editados por Erich Adickes, Friedrich Berger, Gerhard Lehmann y Arthur Buchenau. Finalmente, los volúmenes correspondientes a las clases fueron responsabilidad de Lehmann, Reinhard Brandt y Werner Strack. El monumental proyecto demoró medio siglo en culminarse debido a las dos guerras mundiales y las circunstancias políticas y económicas de Alemania en la primera mitad del siglo XX.

2 Hemos tomado como referencia la carta que Liebert dirige a Gabriel Biguria, en Guatemala, fechada el 17 de junio de 1938.

3 En la página web de la Kant-Gesellschaft (www.kant-gesellschaft.de) puede encontrarse valiosa información histórica acerca de la sociedad. Al año 1910, cuando Liebert se hace cargo de ella contaba con 349 socios, para 1912 la cifra se había casi duplicado, alcanzado a 601. A comienzos de la década de los '20, la Kant-Gesellschaft llegó a tener 3000 integrantes, llegando a ser una de las más importantes sociedades académicas europeas. De otro lado, se indica que Liebert, entre 1918 y 1933 dictó 64 conferencias en filiales en diferentes ciudades alemanas, así como en universidades. Es muy probable que en el marco de sus incansables actividades como conferenciante conociera a Blumenfeld.

4 No solo este trabajo de Blumenfeld fue publicado en la revista de Liebert. También apareció una extensa y muy favorable recensión de su libro Sinn und Unsinn (Blumenfeld, 1933), proveniente de la pluma de Kajica Milanov (1938).

Recibido: 11 de setiembre de 2020

Revisado: 27 de noviembre de $\mathbf{2 0 2 0}$

Aceptado: 20 de febrero de 2021 